\title{
Cyclotron Frequencies and Quanta of the Landau Levels in Semiconductors Having Ellipsoidal Fermi Surfaces Studied in Dependence on Direction of the Magnetic Field
}

\author{
S. OlsZeWski*and T. ROLIŃSKI \\ Institute of Physical Chemistry, Polish Academy of Sciences, M. Kasprzaka 44/52, 01-224 Warsaw, Poland \\ (Received June 23, 2014; in final form November 6, 2014) \\ A method of calculating the Landau levels in crystals having the ellipsoidal Fermi surfaces has been presented \\ with an accent put on the directional dependence of the energy quanta defined by the levels. Physically the \\ problem concerns mainly semiconductors examined in a nearly-free electron approximation. In this case the shape \\ of the Fermi surface is defined by three different effective masses entering the electron Hamiltonian. Beyond of the \\ masses the method, which can be applied for an arbitrary direction of the magnetic field, does contain no empirical \\ parameters in its framework.
}

DOI: 10.12693/APhysPolA.126.1304

PACS: 71.70.Di, 76.40.+b

\section{Introduction}

In general a description and quantization of the energy levels in a system being a free-electron ensemble is well-known. This holds also in the case of presence of the magnetic field acting on the system. However, it is not so easy to calculate the electron motion when the potential of the crystal lattice is combined with the magnetic field. This obstacle is connected with a general difficulty to examine the motion problem when the lattice potential and the magnetic field act simultaneously on an electron; see e.g. [1]. One of simplifying assumptions is that electrons are nearly free in vicinity of the band limits but their effective masses are different for different directions considered in a crystal. Such treatment became especially useful in the case of semiconductors.

In particular Shockley [2] has proposed to examine an ellipsoidal Fermi surface having different effective masses $m_{1}, m_{2}, m_{3}$ in directions along the surface axes [3]. This means the electron energy on the surface is

$$
E=\frac{\hbar^{2}}{2}\left(\frac{k_{x}^{2}}{m_{1}}+\frac{k_{y}^{2}}{m_{2}}+\frac{k_{z}^{2}}{m_{3}}\right) .
$$

Evidently, the positions of the Landau levels as well as the cyclotron frequency for the electron states on the surface should depend on parameters $m_{1}, m_{2}, m_{3}$ entering Eq. (1) as well as the orientation of the magnetic field with respect to the Cartesian coordinate system. The dependences of this kind have been widely examined experimentally on numerous occasions; see e.g. $[4,5]$ for a review. But to the best of our knowledge these calculations represented only the semi-empirical approaches (see e.g. [5-10]) which have never reached an effective theoretical elaboration. The aim of the present paper is to bridge this gap.

${ }^{*}$ corresponding author; e-mail: olsz@ichf .edu.pl

\section{Cyclotron frequency and crystal symmetry}

Certainly a well-known experimental fact of dependence of the cyclotron motion on the orientation of the crystal symmetry species with respect to direction of the magnetic field concerned not only semiconductors.

This property became much evident when the magnetoresistance effect in metals is examined; see [1120]. In fact, both effects, namely the electron circulation and magnetoresistance can be coupled strictly together [11] which explains the importance of the cyclotron frequency for magnetoresistance. More recently the cyclotron motion and magnetoresistance in threedimensional metallic-like systems have been examined rather extensively in [21, 22].

A characteristic property of calculations done on the cyclotron frequency was that they referred to the magnetic field parallel to some symmetry species of the crystal lattice having a high symmetry; see [23, 24]. Our task is to examine first the frequency change due to a change of such highly-symmetric potential. Simultaneously we assume that the structure of the electron states remains rather simple. This means that we allow for the electron states in a crystal do not differ much from the free-electron wave functions.

Our approach is based first on a semiclassical analysis of the electron circulation frequency which is next applied to the Landau levels. Since the cyclotron frequency and the frequency entering the spectrum of the Landau levels are essentially the same, in the first step the cyclotron frequency and its perturbation in some simple systems have been calculated. In a further step the crystals having ellipsoidal Fermi surfaces are mainly considered.

\section{Cyclotron frequency referred to special directions of the magnetic field}

A usual trick in calculating the cyclotron frequency is to assume that the magnetic field is parallel to one of the symmetry crystal axes say that taken along 
the axis $z$. This implies that the cyclotron motion is going on in the planes parallel to the $(x, y)$ plane. In consequence the parts of the crystal Hamiltonian dependent on variables $x$ and $y$ which are normal to the field direction remain active, whereas the variable $z$ parallel to the field behaves like a constant number. A suitable approach can be based on the linear combination of atomic orbitals (LCAO) Hamiltonian applied, for example, to crystals having cubic symmetry [25-27]. In this case the variables $k_{x}, k_{y}$ remain only active in the Hamiltonian represented by a function of $k_{x}, k_{y}$ and $k_{z}$.

For example the $s$ electrons in three lattices of cubic symmetry, i.e. the simple cubic (sc), body-centered cubic (bcc) and face-centered cubic (fcc) can be considered in precisely the same way [28-30]. An important point for further calculations is that all lattices (sc, bcc and fcc) give for small $k_{x}, k_{y}$ and $k_{z}$ the same free-electron energy expression

$$
E=\beta^{\text {latt }} \frac{k_{x}^{2}+k_{y}^{2}+k_{z}^{2}}{2} a_{\text {latt }}^{2},
$$

in which a multiple of the hopping integral

$$
\beta^{\text {latt }}=\beta^{\mathrm{sc}}, \beta^{\mathrm{bcc}}, \beta^{\mathrm{fcc}}
$$

between the $s$-states of the nearest atomic neighbours is taken into account respectively for each lattice case and $a_{\text {latt }}$ is the lattice parameter. But a still more simple expression for energy is obtained when the electron motion in direction $z$ of the magnetic field can be neglected. In this case we have

$$
E^{k_{z}=0}=\beta^{\text {latt }} \frac{k_{x}^{2}+k_{y}^{2}}{2} a_{\text {latt }}^{2} .
$$

The next step is to apply the Wannier idea concerning the action of the Lorentz force on a crystal [31], developed by Suhl [32]. For the magnetic field parallel to $z$ we put for example $k_{x}=x$ for a position coordinate and $k_{y}=p_{x}$ for the corresponding momentum coordinate of the Hamiltonian.

In this case for $\beta^{\text {latt }}=1$ and $a_{\text {latt }}=1$ in (4) the Hamilton equations become

$$
\begin{aligned}
& \frac{\mathrm{d} x}{\mathrm{~d} t}=\frac{\partial E^{k_{z}=0}}{\partial p_{x}}=p_{x}, \\
& \frac{\mathrm{d} p_{x}}{\mathrm{~d} t}=-\frac{\partial E^{k_{z}=0}}{\partial x}=-x,
\end{aligned}
$$

in result of which we obtain

$$
\frac{\mathrm{d}^{2} x}{\mathrm{~d} t^{2}}=\frac{\mathrm{d}}{\mathrm{d} t}\left(\frac{\mathrm{d} x}{\mathrm{~d} t}\right)=\frac{\mathrm{d} p_{x}}{\mathrm{~d} t}=-x .
$$

This is a typical harmonic oscillator with the frequency

$$
\omega_{0}=1 \text {. }
$$

A full cyclotron frequency is obtained by multiplying expression in (8) by the well-known free-electron result

$$
\Omega_{0}=\frac{e B}{m c}
$$

see $[28-30]$.

For semiconductors very many Fermi surfaces can be represented with the aid of a three-parameter energy band (1). If we assume that the magnetic field is acting along the axis $z$, we have instead of (4) the energy

$$
E^{k_{z}=0}=\frac{\hbar^{2}}{2}\left(\frac{k_{x}^{2}}{m_{1}}+\frac{k_{y}^{2}}{m_{2}}\right) .
$$

The components of the Lorentz equation give [3]:

$$
\begin{aligned}
& \hbar \frac{\mathrm{d} k_{x}}{\mathrm{~d} t}=\frac{e}{c} v_{y} B_{z}=\frac{e}{c} \frac{1}{\hbar} \frac{\partial E^{k_{z}=0}}{\partial k_{y}} B_{z}=\frac{e}{c} B_{z} \frac{\hbar k_{y}}{m_{2}}, \\
& \hbar \frac{\mathrm{d} k_{y}}{\mathrm{~d} t}=-\frac{e}{c} v_{x} B_{z}=-\frac{e}{c} \frac{1}{\hbar} \frac{\partial E^{k_{z}=0}}{\partial k_{x}} B_{z}=-\frac{e}{c} B_{z} \frac{\hbar k_{x}}{m_{1}} .
\end{aligned}
$$

In the next step let us put

$$
\begin{aligned}
& \widetilde{k}_{x}=\frac{k_{x}}{\sqrt{m_{1}}}, \\
& \widetilde{k}_{y}=\frac{k_{y}}{\sqrt{m_{2}}} .
\end{aligned}
$$

These substitutions give from (10) and (11) the pair of equations

$$
\begin{aligned}
\frac{\mathrm{d} \widetilde{k}_{x}}{\mathrm{~d} t} & =\frac{e B_{z}}{c \sqrt{m_{1} m_{2}}} \widetilde{k}_{y}, \\
\frac{\mathrm{d} \widetilde{k}_{y}}{\mathrm{~d} t} & =-\frac{e B_{z}}{c \sqrt{m_{1} m_{2}}} \widetilde{k}_{x} .
\end{aligned}
$$

The next differentiation with respect to time can be performed giving, say, for $\widetilde{k}_{x}$ the formula

$$
\frac{\mathrm{d}^{2} \widetilde{k}_{x}}{\mathrm{~d} t^{2}}=\frac{e B_{z}}{c \sqrt{m_{1} m_{2}}} \frac{\mathrm{d} \widetilde{k}_{y}}{\mathrm{~d} t}=-\left(\frac{e B_{z}}{c}\right)^{2} \frac{1}{m_{1} m_{2}} \widetilde{k}_{x} .
$$

Hence we obtain the circular motion represented by a harmonic oscillator having the frequency

$$
\Omega=\frac{e B_{z}}{c} \frac{1}{\left(m_{1} m_{2}\right)^{1 / 2}},
$$

instead of the well-known frequency on the isotropic Fermi surface equal to (9). Evidently, the harmonic oscillator having the frequency (17) can be quantized in a usual way [33] giving separations between the Landau levels equal to

$\hbar \Omega$

instead of separations

$\hbar \Omega_{0}$

obtained with the aid of $\Omega_{0}$ in (9).

\section{Asymmetry of the crystal potential and its effect on the crystal gyration}

A well-known complication connected with any anisotropic Fermi surface is that the electron gyration on such surface changes with any infinitesimal change of direction of the magnetic field. This problem can be also solved on the basis of the Wannier method. For example for a perfect cubic lattice having the tightly-bound $s$ electrons connected with each atom, and for small $k_{x}$, $k_{y}$ and $k_{z}$, three components of energy in (2) are fully equivalent. An asymmetry of the energy expression is obtained when at least one of the components in (2), say that giving energy along axis $z$, is modified, so 


$$
E=\frac{1}{2} \beta^{\text {latt }} a_{\text {latt }}^{2}\left[k_{x}^{2}+k_{y}^{2}+(1+\varepsilon) k_{z}^{2}\right],
$$

where $\varepsilon$ is assumed to be a small number. Physically such change is similar to the change of the free-electron mass $m$ in direction $z$ to an effective mass

$$
m \Rightarrow \frac{m}{1+\varepsilon}
$$

if the fact that $m$ enters the denominator of the free-electron energy expression is taken into account; see e.g. [2, 3].

A practical way to approach the cyclotron gyration frequency in the case of the potential deformation leading to the changed energy (19) is to consider the free-electron motion in the magnetic field tilted to the Cartesian symmetry axes which give the components $k_{x}, k_{y}$ and $k_{z}$. This means that if the magnetic field in the original system of coordinates was along $k_{z}$, a new direction of the magnetic field is parallel to the component $k_{z}^{\prime}$ of vector $\boldsymbol{k}^{\prime}$ defined in a system of the Cartesian coordinates tilted to the original system. A well known reference of the both systems is given by the Euler angles; see e.g. [34].

In a tilted field the electrons gyrate in the planes normal to $k_{z}^{\prime}$, and the value

$$
k_{z}^{\prime}=\text { const }=0
$$

can be chosen as a plane of a special interest. But the energy in expression (2) does not change upon the change of the coordinate system. Therefore, by assuming that in a tilted field the dynamical variables are

$$
\begin{aligned}
& k_{x}^{\prime}=x, \\
& k_{y}^{\prime}=p_{x},
\end{aligned}
$$

the expression (2) with a simplification

$$
\beta^{\text {latt }}=a_{\text {latt }}=1
$$

becomes

$$
\begin{aligned}
& E=\frac{1}{2}\left(k_{x}^{2}+k_{y}^{2}+k_{z}^{2}\right)= \\
& \quad \frac{1}{2}\left[\left(a_{1} x+b_{1} p_{x}\right)^{2}+\left(a_{2} x+b_{2} p_{x}\right)^{2}+\left(a_{3} x+b_{3} p_{x}\right)^{2}\right] .
\end{aligned}
$$

Here the coordinates entering the original energy expression (2) are replaced by

$$
\begin{aligned}
& k_{x}=a_{1} k_{x}^{\prime}+b_{1} k_{y}^{\prime}=a_{1} x+b_{1} p_{x}, \\
& k_{y}=a_{2} k_{x}^{\prime}+b_{2} k_{y}^{\prime}=a_{2} x+b_{2} p_{x}, \\
& k_{z}=a_{3} k_{x}^{\prime}+b_{3} k_{y}^{\prime}=a_{3} x+b_{3} p_{x} .
\end{aligned}
$$

Let us note that only variables $x$ and $p_{x}$ defined in (22) and (23) enter (25) because $k_{z}^{\prime}$ has been eliminated in virtue of the formula (21). The constant terms entering (26)-(28) expressed as functions of the Euler angles are

$$
\begin{aligned}
& a_{1}=\cos \psi \cos \varphi-\cos \vartheta \sin \varphi \sin \psi, \\
& a_{2}=\cos \psi \sin \varphi+\cos \vartheta \cos \varphi \sin \psi, \\
& a_{3}=\sin \vartheta \sin \psi, \\
& b_{1}=-\sin \psi \cos \varphi-\cos \vartheta \sin \varphi \cos \psi, \\
& b_{2}=-\sin \psi \sin \varphi+\cos \vartheta \cos \varphi \cos \psi, \\
& b_{3}=\sin \vartheta \cos \psi .
\end{aligned}
$$

$$
\begin{aligned}
& \frac{\mathrm{d} x}{\mathrm{~d} t}=\frac{\partial E}{\partial p_{x}}= \\
& \quad\left(a_{1} x+b_{1} p_{x}\right) b_{1}+\left(a_{2} x+b_{2} p_{x}\right) b_{2}+\left(a_{3} x+b_{3} p_{x}\right) b_{3} \\
& \frac{\mathrm{d} p_{x}}{\mathrm{~d} t}=-\frac{\partial E}{\partial x}= \\
& \quad-\left(a_{1} x+b_{1} p_{x}\right) a_{1}-\left(a_{2} x+b_{2} p_{x}\right) a_{2}-\left(a_{3} x+b_{3} p_{x}\right) a_{3}
\end{aligned}
$$

They give the oscillator equation

$$
\begin{aligned}
& \frac{\mathrm{d}^{2} x}{\mathrm{~d} t^{2}}=\frac{\mathrm{d}}{\mathrm{d} t}\left(\frac{\mathrm{d} x}{\mathrm{~d} t}\right)=\left(a_{1} b_{1}+a_{2} b_{2}+a_{3} b_{3}\right) \\
& \quad \times\left[\left(a_{1} b_{1}+a_{2} b_{2}+a_{3} b_{3}\right) x+\left(b_{1}^{2}+b_{2}^{2}+b_{3}^{2}\right) p_{x}\right] \\
& -\left(a_{1}^{2}+a_{2}^{2}+a_{3}^{2}\right)\left(b_{1}^{2}+b_{2}^{2}+b_{3}^{2}\right) x \\
& \quad-\left(a_{1} b_{1}+a_{2} b_{2}+a_{3} b_{3}\right)\left(b_{1}^{2}+b_{2}^{2}+b_{3}^{2}\right) p_{x}= \\
& \quad\left[\left(a_{1} b_{1}+a_{2} b_{2}+a_{3} b_{3}\right)^{2}\right. \\
& \left.-\left(a_{1}^{2}+a_{2}^{2}+a_{3}^{2}\right)\left(b_{1}^{2}+b_{2}^{2}+b_{3}^{2}\right)\right] x .
\end{aligned}
$$

Because of the following properties of the coefficients $a_{i}$ and $b_{i}$ :

$$
\begin{aligned}
& a_{1} b_{1}+a_{2} b_{2}+a_{3} b_{3}=0, \\
& a_{1}^{2}+a_{2}^{2}+a_{3}^{2}=1, \\
& b_{1}^{2}+b_{2}^{2}+b_{3}^{2}=1,
\end{aligned}
$$

substituted into (37) we have

$$
\frac{\mathrm{d}^{2} x}{\mathrm{~d} t^{2}}=(0-1 \cdot 1) x=-x .
$$

This is an equation remaining in a full accordance with (7). The formula (41) is an expected result since the free-electron motion in a constant magnetic field is not affected by the choice of the direction of the field.

In the case of a deformed crystal potential along axis $z$ the Hamiltonian (19) takes the form [see (24)]:

$$
E=\frac{1}{2}\left[k_{x}^{2}+k_{y}^{2}+(1+\varepsilon) k_{z}^{2}\right],
$$

therefore only terms containing $a_{i}$ and $b_{i}$ are affected by the perturbation due to $\varepsilon$. Since (42) becomes

$$
\begin{aligned}
E & =\frac{1}{2}\left[\left(a_{1} x+b_{1} p_{x}\right)^{2}+\left(a_{2} x+b_{2} p_{x}\right)^{2}\right. \\
& \left.+(1+\varepsilon)\left(a_{3} x+b_{3} p_{x}\right)^{2}\right],
\end{aligned}
$$

it gives instead of the oscillator in (41) the following result:

$$
\begin{aligned}
& \frac{\mathrm{d}^{2} x}{\mathrm{~d} t^{2}}=\left\{\left[a_{1} b_{1}+a_{2} b_{2}+a_{3} b_{3}(1+\varepsilon)\right]^{2}\right. \\
& \left.\quad-\left[a_{1}^{2}+a_{2}^{2}+(1+\varepsilon) a_{3}^{2}\right]\left[b_{1}^{2}+b_{2}^{2}+(1+\varepsilon) b_{3}^{2}\right]\right\} x= \\
& \quad\left[\left(a_{3} b_{3} \varepsilon\right)^{2}-\left(1+\varepsilon a_{3}^{2}\right)\left(1+\varepsilon b_{3}^{2}\right)\right] x= \\
& \quad\left[-\varepsilon\left(a_{3}^{2}+b_{3}^{2}\right)-1\right] x .
\end{aligned}
$$

In calculating (43) the orthogonality relation (38) together with (39) and (40) has been taken into account.

From (43) we obtain the frequency square

$$
\omega_{\varepsilon}^{2}=1+\varepsilon\left(a_{3}^{2}+b_{3}^{2}\right)=1+\varepsilon \sin ^{2} \vartheta,
$$




$$
\omega_{\varepsilon}=\omega_{\varepsilon}^{(z)} \cong 1+\frac{1}{2} \varepsilon\left(a_{3}^{2}+b_{3}^{2}\right)=1+\frac{1}{2} \varepsilon \sin ^{2} \vartheta
$$

We see that for $\varepsilon>0$ the frequency is increased due to the crystal perturbation. A similar increase concerns the energy in (42).

We can examine the effect of the crystal energy change also in directions $x$ and $y$. Consequently we obtain the frequency

$$
\begin{gathered}
\omega_{\varepsilon}=\omega_{\varepsilon}^{(x)} \approx 1+\frac{1}{2} \varepsilon\left(a_{1}^{2}+b_{1}^{2}\right)= \\
1+\frac{1}{2} \varepsilon\left(\cos ^{2} \varphi+\cos ^{2} \vartheta \sin ^{2} \varphi\right)
\end{gathered}
$$

for direction $x$, and the frequency

$$
\begin{gathered}
\omega_{\varepsilon}=\omega_{\varepsilon}^{(y)} \approx 1+\frac{1}{2} \varepsilon\left(a_{2}^{2}+b_{2}^{2}\right)= \\
1+\frac{1}{2} \varepsilon\left(\sin ^{2} \varphi+\cos ^{2} \vartheta \cos ^{2} \varphi\right)
\end{gathered}
$$

for direction $y$. A characteristic point is that the angular dependences obtained in (45), (46), and (47) are different.

\section{Anisotropy of the energy expressions and its effect on the cyclotron frequency in the case of two-dimensional systems}

For two-dimensional systems the expressions entering the energy in a tilted field may become dependent solely on a single Euler angle instead of their dependence on three angles in a three-dimensional space. For example by assuming

$$
\varphi=0, \quad \psi=0
$$

we obtain:

$$
\begin{aligned}
& a_{1}=1, \\
& a_{2}=0, \\
& a_{3}=0, \\
& b_{1}=0, \\
& b_{2}=\cos \vartheta, \\
& b_{3}=\sin \vartheta .
\end{aligned}
$$

Evidently the coefficients data of the above equations satisfy the orthogonality and normalization conditions of (38)-(40). The frequency squares become

$$
\begin{aligned}
& 1+\varepsilon\left(a_{1}^{2}+b_{1}^{2}\right)=1+\varepsilon, \\
& 1+\varepsilon\left(a_{2}^{2}+b_{2}^{2}\right)=1+\varepsilon \cos ^{2} \vartheta, \\
& 1+\varepsilon\left(a_{3}^{2}+b_{3}^{2}\right)=1+\varepsilon \sin ^{2} \vartheta .
\end{aligned}
$$

But other situations than represented in (48)-(53) can be also examined. These are for $\vartheta=0$ and $\varphi=0$ :

$$
\begin{aligned}
& a_{1}=\cos \psi, \\
& a_{2}=\sin \psi, \\
& b_{1}=-\sin \psi, \\
& b_{2}=\cos \psi, \\
& a_{3}=b_{3}=0,
\end{aligned}
$$

$$
\begin{aligned}
& a_{1}=\cos \varphi, \\
& a_{2}=\sin \varphi, \\
& b_{1}=-\sin \varphi, \\
& b_{2}=\cos \varphi, \\
& a_{3}=b_{3}=0 .
\end{aligned}
$$

The data of (57)-(61) give the following frequency square of the perturbed system:

$$
1+\varepsilon\left(a_{1}^{2}+b_{1}^{2}\right)=1+\varepsilon\left(a_{2}^{2}+b_{2}^{2}\right)=1+\varepsilon
$$
and

$$
1+\varepsilon\left(a_{3}^{2}+b_{3}^{2}\right)=1 .
$$

Similar frequency results independent of the angle coordinate are obtained from (62)-(66).

A characteristic point of (56) is that the effect of deformation of a two-dimensional energy upon the cyclotron frequency is equal to that obtained in three dimensions; see $(44)$.

\section{Cyclotron frequency of electrons gyrating along an ellipsoidal Fermi surface}

The electron energy on such surface is given by the formula (1). This means that the cyclotron frequency on the surface should depend on parameters

$$
m_{1}, m_{2}, m_{3}>0
$$

as well as the orientation of the magnetic field with respect to the Cartesian coordinate system. To the best of our knowledge such a general frequency expression has not been yet calculated and the aim of the present paper is to bridge this gap.

We apply again a tilted system of coordinates for the wave vector $\boldsymbol{k}^{\prime}$ and the magnetic field is directed along the coordinate $k_{z}^{\prime}$ of the tilted system. Assuming that the motion plane normal to the field is

$$
k_{z}^{\prime}=0
$$

we obtain the following expression for $E$ in (69):

$$
\begin{aligned}
E & =\frac{\hbar^{2}}{2}\left[\frac{\left(a_{1} x+b_{1} p_{x}\right)^{2}}{m_{1}}\right. \\
& \left.+\frac{\left(a_{2} x+b_{2} p_{x}\right)^{2}}{m_{2}}+\frac{\left(a_{3} x+b_{3} p_{x}\right)^{2}}{m_{3}}\right] .
\end{aligned}
$$

Here the coordinates $k_{x}, k_{y}, k_{z}$ are obtained in terms of coordinate $(70)$ as well as coordinates

$$
\begin{aligned}
& k_{x}^{\prime}=x, \\
& k_{y}^{\prime}=p_{x},
\end{aligned}
$$

normal to the direction of a tilted field with the aid of the Euler angles; see (26)-(34).

Evidently the energy $E$ in (71) is a non-parabolic expression in the variables $k_{x}^{\prime}$ and and $k_{y}^{\prime}$ defined in (72) and (73), on condition arbitrary values of $a_{1}, a_{2}, a_{3}, b_{1}$, $b_{2}, b_{3}$ as well $m_{1}, m_{2}, m_{3}$ are considered; cf. also Eq. (25). The problem of the electron gyration for such $E$ in the magnetic field directed along an arbitrary axis $k_{z}^{\prime}$ seems to be never solved before.

The electron gyration in the plane $k_{z}^{\prime}=0$ is governed by the equations being components of the Lorentz equation [3]:

and for $\vartheta=0$ and $\psi=0$ : 


$$
\begin{aligned}
& \hbar \frac{\mathrm{d} k_{x}^{\prime}}{\mathrm{d} t}=\hbar \frac{\mathrm{d} x}{\mathrm{~d} t}=\frac{e}{c} v_{y} B=\frac{e}{c} \frac{1}{\hbar} \frac{\partial E}{\partial k_{y}^{\prime}} B, \\
& \hbar \frac{\mathrm{d} k_{y}^{\prime}}{\mathrm{d} t}=\hbar \frac{\mathrm{d} p_{x}}{\mathrm{~d} t}=-\frac{e}{c} v_{x} B=-\frac{e}{c} \frac{1}{\hbar} \frac{\partial E}{\partial k_{x}^{\prime}} B .
\end{aligned}
$$

Here $v_{y}$ is the electron velocity in direction of the axis $k_{y}^{\prime}=p_{x}$ normal to the axis $x$ and $v_{x}$ is directed along the axis $k_{x}^{\prime}=x$; see (72) and (73).

The energy expression in (71) together with (74) and $(75)$ gives

$$
\begin{aligned}
& \hbar \frac{\mathrm{d} x}{\mathrm{~d} t}=\frac{e B}{\hbar c} \hbar^{2} \\
& \quad \times\left(\frac{a_{1} x+b_{1} p_{x}}{m_{1}} b_{1}+\frac{a_{2} x+b_{2} p_{x}}{m_{2}} b_{2}+\frac{a_{3} x+b_{3} p_{x}}{m_{3}} b_{3}\right), \\
& \hbar \frac{\mathrm{d} p_{x}}{\mathrm{~d} t}=-\frac{e B}{\hbar c} \hbar^{2} \\
& \quad \times\left(\frac{a_{1} x+b_{1} p_{x}}{m_{1}} a_{1}+\frac{a_{2} x+b_{2} p_{x}}{m_{2}} a_{2}+\frac{a_{3} x+b_{3} p_{x}}{m_{3}} a_{3}\right) .
\end{aligned}
$$

The next step is the second differentiation of $x$ with respect to time

$$
\begin{aligned}
& \frac{\mathrm{d}^{2} x}{\mathrm{~d} t^{2}}=\frac{\mathrm{d}}{\mathrm{d} t}\left(\frac{\mathrm{d} x}{\mathrm{~d} t}\right)=\frac{e B}{c}\left(\frac{a_{1} b_{1}}{m_{1}}+\frac{a_{2} b_{2}}{m_{2}}+\frac{a_{3} b_{3}}{m_{3}}\right) \frac{\mathrm{d} x}{\mathrm{~d} t} \\
& +\frac{e B}{c}\left(\frac{b_{1}^{2}}{m_{1}}+\frac{b_{2}^{2}}{m_{2}}+\frac{b_{3}^{2}}{m_{3}}\right) \frac{\mathrm{d} p_{x}}{\mathrm{~d} t}=\frac{e B}{c}\left(\frac{a_{1} b_{1}}{m_{1}}\right. \\
& \left.+\frac{a_{2} b_{2}}{m_{2}}+\frac{a_{3} b_{3}}{m_{3}}\right)\left[\frac{e B}{c}\left(\frac{a_{1} b_{1}}{m_{1}}+\frac{a_{2} b_{2}}{m_{2}}+\frac{a_{3} b_{3}}{m_{3}}\right) x\right. \\
& +\frac{e B}{c}\left(\frac{a_{1} b_{1}}{m_{1}}+\frac{a_{2} b_{2}}{m_{2}}+\frac{a_{3} b_{3}}{m_{3}}\right)+\frac{e B}{c}\left(\frac{b_{1}^{2}}{m_{1}}\right. \\
& \left.\left.+\frac{b_{2}^{2}}{m_{2}}+\frac{b_{3}^{2}}{m_{3}}\right) p_{x}\right]+\frac{e B}{c}\left(\frac{b_{1}^{2}}{m_{1}}+\frac{b_{2}^{2}}{m_{2}}+\frac{b_{3}^{2}}{m_{3}}\right) \\
& \quad-\frac{e B}{c}\left(\frac{a_{1}^{2}}{m_{1}}+\frac{a_{2}^{2}}{m_{2}}+\frac{a_{3}^{2}}{m_{3}}\right) x \frac{e B}{c}\left(\frac{a_{1} b_{1}}{m_{1}}+\frac{a_{2} b_{2}}{m_{2}}\right. \\
& \left.\left.+\frac{a_{3} b_{3}}{m_{3}}\right)-\frac{e B}{c}\left(\frac{a_{1} b_{1}}{m_{1}}+\frac{a_{2} b_{2}}{m_{2}}+\frac{a_{3} b_{3}}{m_{3}}\right) p_{x}\right]= \\
& \left(\frac{e B}{c}\right)^{2}\left[\left(\frac{a_{1} b_{1}}{m_{1}}+\frac{a_{2} b_{2}}{m_{2}}+\frac{a_{3} b_{3}}{m_{3}}\right)^{2}\right. \\
& \left.-\left(\frac{a_{1}^{2}}{m_{1}}+\frac{a_{2}^{2}}{m_{2}}+\frac{a_{3}^{2}}{m_{3}}\right)\left(\frac{b_{1}^{2}}{m_{1}}+\frac{b_{2}^{2}}{m_{2}}+\frac{b_{3}^{2}}{m_{3}}\right)\right] x .
\end{aligned}
$$

The result in (78) does not contain the term $p_{x}$ because the dependence on $p_{x}$ is cancelled. A transformation of (78) into the oscillator equation

$$
\frac{\mathrm{d}^{2} x}{\mathrm{~d} t^{2}}+\Omega^{2} x=0
$$

gives the cyclotron frequency square equal to

$$
\begin{aligned}
\Omega^{2} & =\left(\frac{e B}{c}\right)^{2}\left[\left(\frac{a_{1}^{2}}{m_{1}}+\frac{a_{2}^{2}}{m_{2}}+\frac{a_{3}^{2}}{m_{3}}\right)\left(\frac{b_{1}^{2}}{m_{1}}+\frac{b_{2}^{2}}{m_{2}}+\frac{b_{3}^{2}}{m_{3}}\right)\right. \\
& \left.-\left(\frac{a_{1} b_{1}}{m_{1}}+\frac{a_{2} b_{2}}{m_{2}}+\frac{a_{3} b_{3}}{m_{3}}\right)^{2}\right] .
\end{aligned}
$$

The terms $a_{1}, a_{2}, a_{3}$ and $b_{1}, b_{2}, b_{3}$ are calculated in (29)-(34).

\section{Special cases of the cyclotron frequency on the ellipsoidal Fermi surface}

Very simple expressions for the cyclotron frequency concern special situations of the magnetic field, for example those represented by the parameter values

$$
\begin{array}{ll}
a_{1}=1, & b_{2}=1, \\
a_{2}=1, & b_{3}=1, \\
a_{3}=1, & b_{1}=1,
\end{array}
$$

The above formulae imply the relations

$$
\begin{aligned}
& a_{2}=a_{3}=b_{1}=b_{3}=0, \\
& a_{1}=a_{3}=b_{1}=b_{2}=0, \\
& a_{1}=a_{2}=b_{2}=b_{3}=0,
\end{aligned}
$$

valid respectively for (81)-(83); see (26)-(28) as well as (38)-(40). On the basis of (29)-(34) we should have

$$
\psi=\varphi=\vartheta=0
$$

for the case of (81) and (84),

$$
\psi=0, \quad \varphi=\frac{\pi}{2}, \quad \vartheta=\frac{\pi}{2}
$$

for the case of (82) and (85),

$$
\psi=-\frac{\pi}{2}, \quad \varphi=0, \quad \vartheta=-\frac{\pi}{2}
$$

for the case of (83) and (86).

The frequency square in (80) becomes

$$
\Omega^{2}=\left(\frac{e B}{c}\right)^{2} \frac{1}{m_{1} m_{2}}
$$

in correspondence to (81), (84), and (87),

$$
\Omega^{2}=\left(\frac{e B}{c}\right)^{2} \frac{1}{m_{2} m_{3}}
$$

in correspondence to (82), (85), and (88),

$$
\Omega^{2}=\left(\frac{e B}{c}\right)^{2} \frac{1}{m_{3} m_{1}}
$$

in correspondence to (83), (86), and (89).

The frequencies (90)-(92) are compatible with the special situations of the magnetic field discussed in Sect. 3.

\section{Directional dependence of the energy quanta defined by the Landau levels}

The Euler angles entering parameters $a_{1}, a_{2}, a_{3}$ and $b_{1}, b_{2}, b_{3}$ in (80) should not be considered on an equal footing. In fact the electron circulation in the magnetic field put along direction $z^{\prime}$ of the Cartesian coordinate system $\left(x^{\prime}, y^{\prime}, z^{\prime}\right)$ tilted to the original system $(x, y, z)$ is connected with a rapid change of the angle $\psi$. A rapid pulsation of $\psi$ is accompanied by a constant behaviour of $\vartheta$ and $\varphi$. Therefore a good approximation to the motion dependence on the angles $\vartheta$ and $\varphi$ can be obtained by assuming the averaged expressions

$$
\overline{\sin ^{2} \psi}=\overline{\cos ^{2} \psi}=\frac{1}{2},
$$

$\overline{\sin \psi \cos \psi}=0$, 

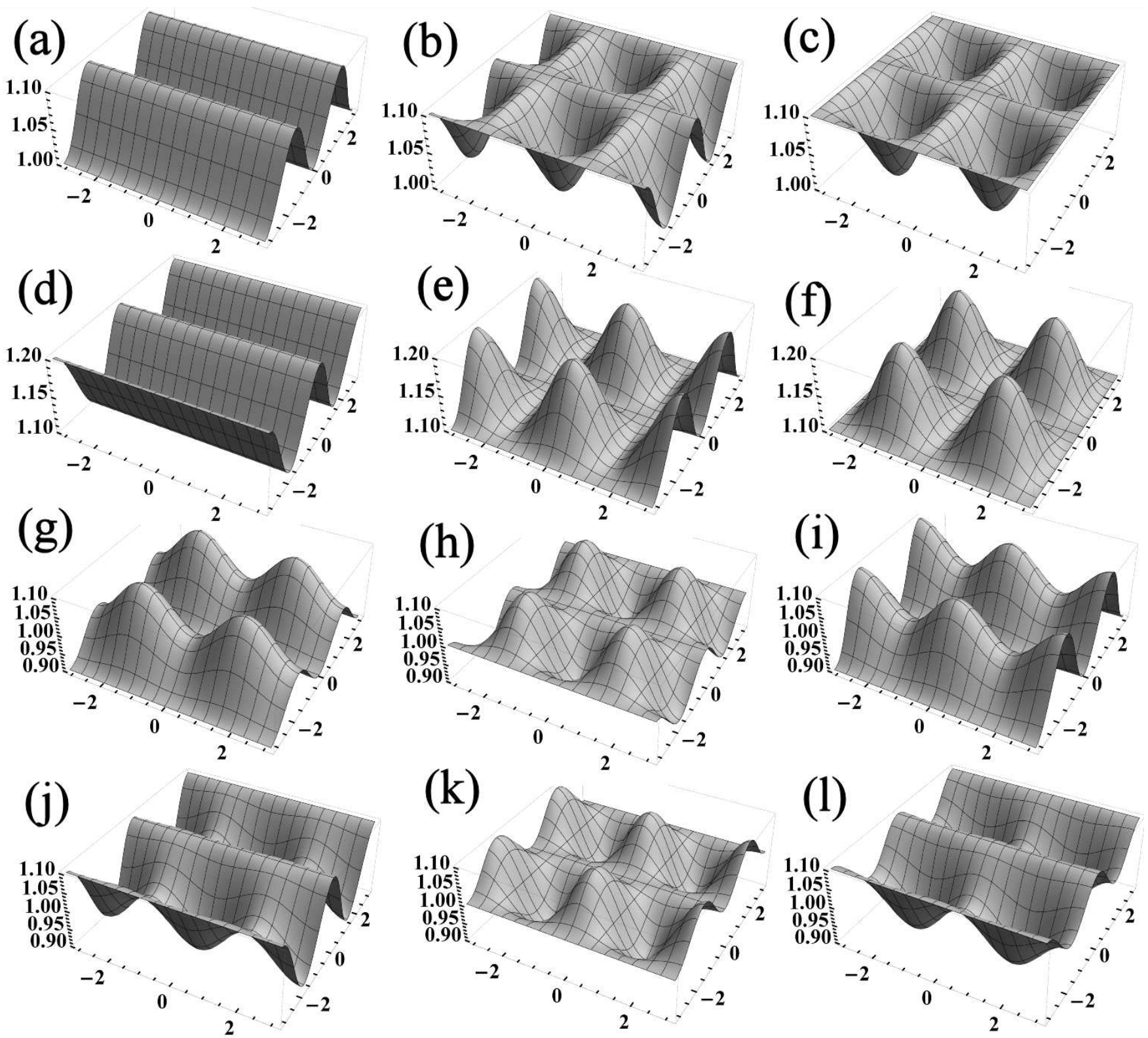

Fig. 1. $F$ in (107) plotted versus $\varphi$ and $\vartheta$ : (a) $m_{1} / m=m_{2} / m=1, m_{3} / m=5 / 6$; (b) $m_{1} / m=1, m_{2} / m=5 / 6$, $m_{3} / m=1$; (c) $m_{1} / m=5 / 6, m_{2} / m=m_{3} / m=1$; (d) $m_{1} / m=m_{2} / m=5 / 6, m_{3} / m=1$; (e) $m_{1} / m=5 / 6, m_{2} / m=1$, $m_{3} / m=5 / 6$; (f) $m_{1} / m=1, m_{2} / m=m_{3} / m=5 / 6$; (g) $m_{1} / m=5 / 4, m_{2} / m=1, m_{3} / m=5 / 6$; $(\mathrm{h}) m_{1} / m=5 / 4$, $m_{2} / m=5 / 6, m_{3} / m=1$; (i) $m_{1} / m=1, m_{2} / m=5 / 4, m_{3} / m=5 / 6$; (j) $m_{1} / m=1, m_{2} / m=5 / 6, m_{3} / m=5 / 4$; (k) $m_{1} / m=5 / 6, m_{2} / m=5 / 4, m_{3} / m=1$; (l) $m_{1} / m=5 / 6, m_{2} / m=1, m_{3} / m=5 / 4$.

$a_{1}^{2}, a_{2}^{2}, a_{3}^{2}, \quad b_{1}^{2}, b_{2}^{2}, b_{3}^{2}$

as well as

$$
a_{1} b_{1}, \quad a_{2} b_{2} \quad a_{3} b_{3}
$$

which are present in (80). Consequently we obtain from (93) and (94) the averaged expressions

$$
\begin{aligned}
& \bar{a}_{1}^{2}=\frac{1}{2} \cos ^{2} \varphi+\frac{1}{2} \cos ^{2} \vartheta \sin ^{2} \varphi, \\
& \bar{a}_{2}^{2}=\frac{1}{2} \sin ^{2} \varphi+\frac{1}{2} \cos ^{2} \vartheta \cos ^{2} \varphi, \\
& \bar{a}_{3}^{2}=\frac{1}{2} \sin ^{2} \vartheta,
\end{aligned}
$$

$$
\begin{aligned}
& \bar{b}_{1}^{2}=\frac{1}{2} \cos ^{2} \varphi+\frac{1}{2} \cos ^{2} \vartheta \sin ^{2} \varphi, \\
& \bar{b}_{2}^{2}=\frac{1}{2} \sin ^{2} \varphi+\frac{1}{2} \cos ^{2} \vartheta \cos ^{2} \varphi, \\
& \bar{b}_{3}^{2}=\frac{1}{2} \sin ^{2} \vartheta, \\
& \overline{a_{1} b_{1}}=-\frac{1}{2} \cos \varphi \sin \varphi \cos \vartheta+\frac{1}{2} \cos \varphi \sin \varphi \cos \vartheta=0, \\
& \overline{a_{2} b_{2}}=\frac{1}{2} \cos \varphi \sin \varphi \cos \vartheta-\frac{1}{2} \sin \varphi \cos \varphi \cos \vartheta=0, \\
& \overline{a_{3} b_{3}}=0 .
\end{aligned}
$$

In this way the following averaged expression for the frequency square is derived: 


$$
\begin{aligned}
\bar{\Omega}^{2} & =\left(\frac{e B}{c}\right)^{2}\left[\frac{\cos ^{2} \varphi+\cos ^{2} \vartheta \sin ^{2} \varphi}{2 m_{1}}\right. \\
& \left.+\frac{\sin ^{2} \varphi+\cos ^{2} \vartheta \cos ^{2} \varphi}{2 m_{2}}+\frac{\sin ^{2} \vartheta}{2 m_{3}}\right]^{2}=\left(\frac{e B}{m c}\right)^{2} F^{2}
\end{aligned}
$$

where

$$
\begin{aligned}
F & =\left(\cos ^{2} \varphi+\cos ^{2} \vartheta \sin ^{2} \varphi\right) \frac{m}{2 m_{1}} \\
& +\left(\sin ^{2} \varphi+\cos ^{2} \vartheta \cos ^{2} \varphi\right) \frac{m}{2 m_{2}}+\sin ^{2} \vartheta \frac{m}{2 m_{3}} .
\end{aligned}
$$

It is easy to demonstrate that extrema of the expression (107) for $F$ occur at

$$
\begin{aligned}
& 2 \vartheta= \pm n \pi, \\
& 2 \varphi= \pm n^{\prime} \pi
\end{aligned}
$$

where $n$ and $n^{\prime}$ are integer numbers. The dependence of $F$ on $\varphi$ and $\vartheta$ is illustrated in Fig. 1 (a)-(l) for different combinations of parameters

$$
\frac{m_{1}}{m}, \quad \frac{m_{2}}{m}, \quad \frac{m_{3}}{m} .
$$

The axes pointing to the left are those of $\varphi$, the axes pointing to the right are those of $\vartheta$; the angles $\varphi$ and $\vartheta$ are presented in radians. In effect the quanta of energy separating the neighbouring Landau levels are

$$
\Delta E=\hbar(e B / m c) F(\vartheta, \varphi)
$$

where the dependence on $\vartheta$ and $\varphi$, as well as the ratios of the effective masses in (69) to the free-electron mass $m$ enters solely the coefficient $F$; see (107).

\section{Remark on quantization technique of the Landau levels}

A basic result of the paper concerning quantization of the Landau levels are the harmonic oscillator equations which represent a classical approximation of the cyclotron motion. This result holds for all oscillator cases examined in the paper including the electron motion on the ellipsoidal Fermi surfaces characteristic for semiconductors. In effect only one parameter of the motion, i.e. the frequency of the oscillator, depends on such parameters like the effective electron masses as well the Euler angles representing the direction of the magnetic field with respect to the symmetry species of the crystal lattice. In consequence, the directional influence of the magnetic field on the motion is incorporated in the cyclotron frequency alone.

Accordingly, the Landau levels in all examined cases should be represented by the equidistant numbers being multiples of the cyclotron frequency which varies in dependence on the masses $m_{1}, m_{2}, m_{3}$ and the Euler angles. In other words, any set of the effective masses considered on a given Fermi surface and a set of the Euler angles defining the direction of the magnetic field, give a corresponding set of the equidistant Landau levels.

This behavior is in fact confirmed on the experimental basis $[5,9,10]$ for numerous crystal cases excepting for a rather small change of the distances observed between the lowest Landau levels entering a given set of levels, a property which cannot be explained by the present theory.
Since the approach to the electron motion is essentially semiclassical and not quantum-mechanical in its nature, there is no attempt done in the paper to present the effects connected with the electron spin or the spin-orbit coupling considered e.g. in $[4,5]$.

\section{Summary}

A strong dependence of positions of the Landau levels on the direction of the magnetic field in crystals having non-spherical Fermi surfaces is a well-known experimental fact. On the other side, any theoretical approach to this dependence problem has been usually considered to be a difficult task.

The aim of the paper was to present a theory of the effect mentioned above on the basis of a formalism developed a time ago by Wannier [31]. This author has noticed first a property of similarity between the components of the Lorentz equation describing the motion of the crystal electrons in the magnetic field and the Hamilton equations. In the paper we show that a crucial role in calculations can be ascribed to the frequency of the harmonic oscillator which is an effect of superposition of the mentioned Hamilton equations.

This simple kind of the oscillator holds usually on condition when the energy of a crystal electron can be expressed as a quadratic form of the components of the electron wave vector, but this situation is changed into a complicated non-parabolic Hamiltonian (71) valid in case an arbitrary direction of the magnetic field is considered.

A quantization of the oscillator performed in a wellknown way provides us readily with a set of the equidistant Landau levels characteristic for a given direction of the magnetic field. The level positions depend also on the mass parameters defining the shape of the Fermi surface.

\section{References}

[1] G. Watson, Contemp. Phys. 37, 127 (1996).

[2] W. Shockley, Phys. Rev. 90, 491 (1953).

[3] J.C. Slater, Quantum Theory of Molecules and Solids, Vol. 3, McGrawHill, New York 1967.

[4] I.M. Tsidilkovskii, Electrons and Holes in Semiconductors, Nauka, Moscow 1972 (in Russian).

[5] B. Lax, J.G. Mavroides, Solid State Physics, Vol. 11, Eds. F. Seitz, D. Turnbull, Academic, New York 1960, p. 261.

[6] G. Dresselhaus, A.F. Kip, C. Kittel, Phys. Rev. 92, 827 (1953).

[7] R.N. Dexter, B. Lax, Phys. Rev. 96, 223 (1954).

[8] R.N. Dexter, H.J. Zeiger, B. Lax, Phys. Rev. 104, 637 (1956).

[9] S. Zwerdling, B. Lax, L.M. Roth, K.J. Button, Phys. Rev. 114, 80 (1959).

[10] L.M. Roth, B. Lax, S. Zwerdling, Phys. Rev. 114, 90 (1959).

[11] C. Kittel, Quantum Theory of Solids, 2nd ed., Wiley, New York 1987.

[12] N.E. Aleseevskii, Yu.P. Gaidukov, Sov. Phys.-JETP 8, 383 (1958). 
[13] N.E. Aleseevskii, Yu.P. Gaidukov, Sov. Phys.-JETP 9, 311 (1959).

[14] N.E. Aleseevskii, Yu.P. Gaidukov, Sov. Phys.-JETP 10, 481 (1959).

[15] Yu.P. Gaidukov, Sov. Phys.-JETP 10, 913 (1959).

[16] F. Garcia-Moliner, S. Simons, Proc. Phys. Soc. (London) 72, 996 (1958).

[17] E. Justi, Phys. Zeitschr. 41, 563 (1940).

[18] E. Grueneisen, H.D. Erfling, Ann. Phys. 38, 399 (1940).

[19] E. Fawcett, Adv. Phys. 13, 139 (1964).

[20] J.L. Olsen, Electron Transport in Metals, Interscience, New York 1962.

[21] S. Olszewski, Rev. Theor. Sci. 1, 344 (2013).

[22] A.D. Mirlin, J. Wilke, F. Evers, D.G. Polyakov, P. Wölfle, Phys. Rev. Lett. 83, 2801 (1999).

[23] J.M. Luttinger, W. Kohn, Phys. Rev. 97, 869 (1955).
[24] J.M. Luttinger, Phys. Rev. 102, 1030 (1956).

[25] N.F. Mott, H. Jones, Theory of the Properties of Metals and Alloys, Clarendon Press, Oxford 1958.

[26] D.C. Mattis, The Theory of Magnetism Made Simple, World Scientific, Hackensack, New Jersey (USA) 2005.

[27] J.R. Reitz, in: Solid State Physics, Vol. 1, Eds. F. Seitz, D. Turnbull, Academic, New York 1955.

[28] S. Olszewski, T. Roliński, T. Kwiatkowski, Phys. Rev. B 59, 3740 (1999).

[29] S. Olszewski, T. Roliński, Symmetry 3, 541 (2011).

[30] S. Olszewski, Quantum Matter 3, 65 (2014).

[31] G.H. Wannier, Rev. Mod. Phys. 34, 645 (1962).

[32] H. Suhl, J. Phys. (France) 50, 2613 (1989).

[33] L. Landau, Zeits. Physik 64, 629 (1930).

[34] H. Goldstein, Classical Mechanics, Addison-Wesley, Reading (MA) 1965. 